\title{
Cosmic perturbations, baryon asymmetry, and dark matter from the minimal supersymmetric standard model
}

\author{
Keisuke Harigaya \\ School of Natural Sciences, Institute for Advanced Study, Princeton, New Jersey 08540, USA \\ Masaki Yamada® \\ Institute of Cosmology, Department of Physics and Astronomy, Tufts University, \\ Medford, Massachusetts 02155, USA
}

(Received 31 July 2019; revised 10 July 2020; accepted 4 November 2020; published 1 December 2020)

\begin{abstract}
Scalar fields in the minimal supersymmetric standard model may have large field values during inflation. Because of approximate global symmetry, it is plausible that the phase directions of them are nearly massless during inflation and obtain quantum fluctuations, which may be the origin of the cosmic perturbations. If perturbations are produced through Q-ball formation, baryon asymmetry and dark matter can be consistently generated. Significant baryon and dark matter isocurvature perturbations are produced, but they are predicted to nearly compensate each other. The lepton asymmetry is much larger than the baryon asymmetry. The scenario predicts local non-Gaussianity of $f_{\mathrm{NL}}=5 / 3$. The implication to the mass spectrum of supersymmetric particles is discussed.
\end{abstract}

DOI: 10.1103/PhysRevD.102.121301

\section{INTRODUCTION}

The Universe starts from small fluctuations, which have grown and collapsed to form galaxies. The origin of the fluctuations is an elementary question in cosmology and particle physics. The observations of the large scale structure and the cosmic microwave wave background (CMB) have revealed that the fluctuations are nearly Gaussian and scale invariant [1,2], which are naturally explained by quantum fluctuations of a nearly massless scalar field generated during inflation [3-7]. Almost nothing is known about the scalar field. It may be the inflaton itself or another scalar field (e.g., a curvaton [810]) may be as heavy as $10^{13} \mathrm{GeV}$ or, in principle, as light as $10^{-24} \mathrm{GeV}$.

In this paper, we investigate the possibility that scalar fields in the minimal supersymmetric standard model (MSSM) are responsible for the fluctuations. The MSSM is one of the best-motivated extensions of the standard model. It achieves successful precise gauge coupling unification [11-16], provides a dark matter (DM) candidate as the lightest supersymmetric particle (LSP) [17-19], and can explain the electroweak scale much below the unification scale $\sim 10^{16} \mathrm{GeV}[17,20-22]$. In the MSSM, there exist many combinations of scalar fields called flat

Published by the American Physical Society under the terms of the Creative Commons Attribution 4.0 International license. Further distribution of this work must maintain attribution to the author(s) and the published article's title, journal citation, and DOI. Funded by SCOAP ${ }^{3}$. directions, whose potentials vanish at the renormalizable level and supersymmetric limit. It is natural to ask if the cosmic perturbations originate from quantum fluctuations of one of those flat directions [23-28] and seek experimental signatures of such a scenario.

With supersymmetry broken during inflation, flat directions in general obtain soft masses called Hubble induced masses. Suppose a flat direction $\phi$ obtains a negative Hubble-induced mass squared and is destabilized during inflation $[29,30]$. The flat direction is stabilized by a higher dimensional term in the superpotential $W=\lambda \phi^{n}$. The potential of $\phi$ is

$$
V \sim-H^{2}|\phi|^{2}+\lambda^{2}|\phi|^{2 n-2},
$$

so that the field value of $\phi$ during inflation is $\phi_{\text {inf }} \sim\left(H_{\text {inf }} / \lambda\right)^{1 /(n-2)}$, where $H_{\text {inf }}$ is the Hubble scale during inflation. The radial direction has a mass as large as the Hubble scale, and its quantum fluctuations are suppressed. A special case of nearly vanishing Hubble induced masses is discussed in [23-26]. The scalar potential of $\phi$ in Eq. (1) possesses an $U(1)$ symmetry (which is an $\mathrm{R}$ symmetry). It is plausible that even if other possible higher dimensional operators are included, the $U(1)$ symmetry is approximately maintained. The phase direction of $\phi$, which we denote as $\theta$, is then almost massless and obtains quantum fluctuations $\delta \theta \sim H_{\text {inf }} /\left(2 \pi \phi_{\text {inf }}\right)$ during inflation. We investigate if the fluctuations of the phase direction alone can explain the cosmic perturbations. This scenario is examined in [27], neglecting important 
dissipation effects explained below, while [28] predicts non-Gaussianity that is too large.

After inflation, the inflaton, which is different from $\phi$, begins oscillation. We assume that the sign of the Hubble induced mass squared of $\phi$ remains negative. The field value of $\phi$ tracks the minimum, $\phi \sim(H / \lambda)^{1 /(n-2)}$ [29-31]. When the Hubble scale drops below the soft mass of $\phi$ at the vacuum, $m_{\phi}$, the flat direction begins oscillation around the origin. At this point, the irreducible explicit $U(1)$ symmetry breaking from the gravitino mass $m_{3 / 2}$,

$$
\Delta V \sim m_{3 / 2} \lambda \phi^{n}+\text { H.c. },
$$

which gives a nonzero potential energy of $\theta$, is no more negligible. The fluctuation of $\theta$, together with its potential, gives rise to the fluctuation of the energy density and hence, may source the cosmic perturbations [27,28]. This seems challenging for a MSSM flat direction, which has $O(1)$ gauge couplings. Such a field is rapidly dissipated into thermal plasma [32] and never dominates the Universe. If the curvature perturbation of the Universe originates from fluctuations of a subdominant component, the fluctuations must be large, and the resultant perturbation is highly non-Gaussian [33], which is incompatible with the observations [2].

The rapid dissipation is due to a passage of the field near the origin, which is avoided if $\phi$ circulates by a kick from large enough $\Delta V$. The circular motion corresponds to global charge asymmetry of $\phi$. It is tempting to identify the charge asymmetry with baryon asymmetry [29,30], but baryon asymmetry that is too large is produced if $\phi$ dominates the Universe. Moreover, since the energy of the phase direction is comparable to or smaller than that of the radial direction, a baryon isocurvature perturbation that is too large is produced [33].

These problems are evaded if Q-balls form. The radial directions of $\phi$ may have a potential shallower than a quadratic one. Then as $\phi$ oscillates, it develops instability, and nontopological solitons called Q-balls are formed [3441], into which the energy of the phase direction is converted. The dissipation rate of Q-balls is limited by Pauli-blocking near the surface of Q-balls [42]. Q-balls are more long-lived than the radial direction and can dominate the Universe. If the Q-balls have vanishing baryon charges but nonzero lepton charges, an appropriate amount of baryon asymmetry is generated from partial decay of the Q-balls before the electroweak phase transition and sphaleron transitions [43].

In the following, we describe the detail of the scenario as well as its cosmological, astrophysical and collider signatures.

\section{CURVATURE PERTURBATIONS FROM Q-BALLS}

When $\phi$ begins oscillation, the explicit breaking $\Delta V$ drives the angular motion of $\phi$ and produces the global charge asymmetry of $\phi$ [44],

$$
n_{\phi} \sim \epsilon m_{\phi} \phi_{\mathrm{osc}}^{2}, \quad \epsilon \equiv \theta_{\mathrm{osc}} \frac{m_{3 / 2}}{m_{\phi}} .
$$

The field value of $\theta$ is defined so that $\theta=0$ is the minimum of the potential $\Delta V$. The subscript "osc" represents the value at the beginning of the oscillation. Since $\theta$ fluctuates, the charge asymmetry also fluctuates. The asymmetry is approximately conserved afterward since $\Delta V$ becomes negligible as the amplitude of the oscillation decreases by the cosmic expansion. Once the Q-balls are formed, most of the asymmetry is stored in them. As we will see, $\epsilon$ not much below unity is required. This is naturally the case if the potential of $\phi$ around the field value $\phi_{\text {osc }}$ is dominantly given by gravity mediation,

$$
V(\phi)=m_{\phi}^{2}|\phi|^{2}\left(1+K \ln \frac{|\phi|^{2}}{m_{\mathrm{pl}}^{2}}\right),
$$

with $m_{\phi} \sim m_{3 / 2}$. The logarithmic term comes from the renormalization running of the soft mass, and $K$ is negative if the renormalization is dominated by that from gauginos or that from yukawa couplings to scalars with the negative soft mass squared. $|K|=O(0.1-0.01)$ if soft masses are of the same order, while can be as small as $10^{-4}$ if gaugino masses and/or negative soft masses are much smaller than $m_{\phi}$ at a high energy scale. The following computation is also applicable to gauge mediation as long as the potential energy is dominated by gravity mediated one around $\phi=\phi_{\text {osc }}$.

The number density of the Q-balls is determined by the instability scale $\sim|K|^{-1 / 2} m_{\phi}^{-1}$, almost independent of $\theta_{\text {osc }}$ [45], and does not fluctuate. The charge $Q$ and the mass $m_{Q}$ of individual Q-balls (averaged in each Hubble patch) fluctuate as they depend on $\theta_{\text {osc }}$ via $\epsilon$ [37,45-47],

$$
Q \simeq 0.02 \epsilon\left(\frac{|K|}{0.1}\right)^{1 / 2}\left(\frac{\phi_{\mathrm{osc}}}{m_{\phi}}\right)^{2}, \quad m_{Q} \simeq m_{\phi} Q
$$

where we assume $\epsilon \gtrsim 0.01$. We comment on the case with smaller $\epsilon$ as well as a subtle issue for $\epsilon \sim 1$ later. This leads to the fluctuations of the energy density $\rho_{Q}$ and the decay rate $\Gamma_{Q}$ of the Q-balls,

$\rho_{Q}(a) \simeq \epsilon m_{\phi}^{2} \phi_{\mathrm{osc}}^{2}\left(\frac{a_{\mathrm{osc}}}{a}\right)^{3}, \quad \Gamma_{Q} \simeq g \frac{R^{2} m_{\phi}^{3}}{24 \pi Q}\left(\propto \epsilon^{-1}\right)$,

where $R \sim 7 / \sqrt{|K|} m_{\phi}$ is the typical size of the Q-balls and $g(\sim 100)$ is the number of degrees of freedom that couples to the Q-balls $[42,48]$. The decay rate of Q-balls is suppressed by the Pauli-blocking effect, guaranteeing the long lifetime of Q-balls.

At the time of production, the Q-balls are subdominant component of the Universe. They are long-lived, eventually dominate the Universe, and then decay into standard model particles at a temperature, 


$$
\begin{aligned}
T_{\mathrm{dec}} & \simeq\left(\frac{10}{\pi^{2} g_{*}\left(T_{\mathrm{dec}}\right)}\right)^{1 / 4} \sqrt{\Gamma_{Q} m_{\mathrm{pl}}} \\
& \simeq 3.6 \mathrm{GeV}\left(\frac{m_{\phi}}{10 \mathrm{TeV}}\right)^{1 / 2}\left(\frac{1.3 \times 10^{24}}{Q}\right)^{1 / 2} \propto \epsilon^{-1 / 2}
\end{aligned}
$$

where $g_{*}$ is the effective number of relativistic particles and we take $|K|=0.01$. The decay rate and the energy density of the Q-balls are modulated by the fluctuation of $\epsilon$ (or $\left.\theta_{\text {osc }}\right)$, which sources the curvature perturbation.

We compute the magnitude of the curvature perturbation by the $\delta N$ formalism [49-51] with the following history. After inflation and reheating complete, (1) $\phi$ begins oscillation. Soon after that, the instability occurs, and Q-balls form. (2) The Q-balls eventually dominate the Universe and (3) decay afterward at a temperature $T_{\text {dec }}$. (4) The Universe reaches a reference temperature $T_{f}$. It is also possible that $\phi$ begins oscillation before the completion of the reheating, but the property of the produced curvature perturbation remains the same.

We parametrize the energy density of the Q-balls at the beginning of the oscillation as $\rho \epsilon$, and the decay rate of them as $\Gamma / \epsilon$. Numbers of $e$-foldings between the stages (1)-(4) are

$N_{12} \simeq \ln \frac{m_{\phi}^{2} m_{\mathrm{pl}}^{2}}{\rho \epsilon}, \quad N_{23} \simeq \frac{1}{3} \ln \frac{\rho \epsilon}{\Gamma^{2} m_{\mathrm{pl}}^{2} / \epsilon^{2}}$,

$N_{34} \simeq \frac{1}{4} \ln \frac{\Gamma^{2} m_{\mathrm{pl}}^{2} / \epsilon^{2}}{T_{f}^{4}}$.

The total number of $e$-foldings is then

$$
N_{\mathrm{tot}}=-\frac{1}{2} \ln \epsilon+\text { const. }
$$

To the first order in $\delta \epsilon$, the curvature perturbation produced by the Q-balls is

$$
\zeta=\delta N=-\frac{1}{2} \frac{\delta \epsilon}{\epsilon} .
$$

The parameter $\epsilon$ is proportional to the field value of $\theta$. The observed curvature perturbation $\mathcal{P}_{\zeta} \simeq 3 \times 10^{-10}$ [52] requires that

$$
H_{\mathrm{inf}} \simeq 1 \times 10^{13} \mathrm{GeV} \frac{\phi_{\mathrm{inf}}}{10^{17} \mathrm{GeV}}
$$

The contribution to the curvature perturbation through the modulation of the decay rate of the Q-balls is similar to the modulated reheating scenario [53,54]. In our scenario, the decay rate is modulated by the property of the Q-balls themselves without spectator fields.
The spectral index is given by $[49,55]$

$$
n_{s}=1-2 \epsilon_{\mathrm{inf}}+2 \eta_{\theta},
$$

where $\epsilon_{\text {inf }}$ is the first slow-roll parameter of inflation and $\eta_{\theta}$ $\left(=m_{\theta}^{2} / H_{\text {inf }}^{2}\right)$ is determined by the mass of the phase direction $m_{\theta}$. The red-tilted spectrum $n_{s}=0.96-0.98$ requires $\epsilon_{\text {inf }}=$ $O\left(10^{-2}\right)$ or $m_{\theta}^{2}=-O\left(10^{-2}\right) H_{\mathrm{inf}}^{2}$. The former requires a large field inflation [56], while the latter requires the explicit breaking of the approximate $U(1)$ symmetry. In fact, if $\phi_{\text {inf }}$ is close to the cutoff scale, we expect that some higher dimensional terms in the Kahler potential or superpotential are not negligible and give $\theta$ a mass not much below the Hubble scale. For example, a Kahler potential $K \sim \phi^{\ell}$ gives a potential $\sim H^{2} \phi^{\ell} / m_{\mathrm{pl}}^{\ell-2}+$ c.c., where $\ell(\geq 2)$ is an integer depending on flat directions. If $\ell=4$ and $\phi_{\text {inf }} \simeq 10^{17} \mathrm{GeV}$, this term leads to $m_{\theta}^{2}=-O\left(10^{-2}\right) H_{\text {inf }}^{2}$.

The local non-Gaussianity is parametrized by $f_{\mathrm{NL}}$,

$$
\zeta=\zeta_{g}+\frac{3}{5} f_{\mathrm{NL}} \zeta_{g}^{2}
$$

where $\zeta_{g}$ is a Gaussian perturbation. To the second order in $\delta \epsilon$, the curvature perturbation is

$$
\zeta=-\frac{1}{2} \frac{\delta \epsilon}{\epsilon}+\frac{(\delta \epsilon)^{2}}{4 \epsilon^{2}} .
$$

By comparing Eq. (13) with (14), we obtain

$$
f_{\mathrm{NL}}=\frac{5}{3}
$$

which may be detected by future observations of galaxy distributions [57,58] or $21 \mathrm{~cm}$ lines [59].

If $\epsilon \lesssim 0.01$, Q-balls and anti-Q-balls of the almost same charges and energy densities are produced, invalidating Eq. (5) $[45,46,60]$. The fluctuation of $\theta$ only perturbs the charges and number densities of the subdominant component of the Q-balls, producing non-Gaussianity that is too large.

We have implicitly assumed that the dynamics of the radial direction is independent of $\epsilon$, which is the case for $\epsilon<1$. We expect that the dynamics is affected for $\epsilon \sim 1$ as the potential energy of the phase direction is comparable to that of the radial direction. For example, the beginning of oscillation as well as the size of Q-balls may depend on $\epsilon$, giving $O(\epsilon)$ corrections to above formulas. For simplicity, we assume $\epsilon$ is not close to unity and leave investigation of $\epsilon \sim 1$ for future works.

\section{BARYON ASYMMETRY}

If the Q-balls do not have baryon nor lepton charges, or have a vanishing $B-L$ charge and decay before the electroweak phase transition, they do not produce baryon 
asymmetry. Baryon asymmetry can be produced after the Q-balls decay at a low temperature. Possible scenarios include the electroweak baryogenesis [61,62] and the baryogenesis from neutrino oscillation [63]. No baryon isocurvature perturbation is produced in these cases.

The minimal and more interesting possibility is that the Q-balls produce baryon asymmetry. If all the amount of asymmetries in the Q-balls are released into baryons via the decay of the Q-balls, too much baryon asymmetry is produced. We instead consider Q-balls with vanishing baryon charges but nonzero lepton charges $q_{L}$ (i.e., L-balls), like $L L \bar{e}, Q \bar{d} L, Q \bar{u} Q \bar{u} \bar{e}$ flat directions. We consider the case where they decay after the electroweak phase transition so that only leptons emitted from the Q-balls before the electroweak phase transition are converted into baryons by sphaleron transitions [43]. Assuming that the thermal bath is dominated by particles produced by the Q-balls rather than the inflaton produced ones, the amount of the baryon asymmetry $n_{b}$ normalized by the entropy density $s$ is

$$
\begin{aligned}
\frac{n_{b}}{s} & \left.\simeq \frac{28}{79}\left|q_{L}\right| \frac{Q \Gamma_{Q} n_{Q}(t) t}{4 \rho_{Q}(t) / 3 T_{\mathrm{dec}}}\right|_{T=T_{\mathrm{sph}}} \\
& \simeq 9 \times 10^{-11}\left|q_{L}\right|\left(\frac{T_{\mathrm{dec}}}{3.6 \mathrm{GeV}}\right)^{5} \frac{10 \mathrm{TeV}}{m_{\phi}},
\end{aligned}
$$

where $T_{\text {sph }}(\simeq 130 \mathrm{GeV})$ is the sphaleron decoupling temperature [64]. The result explains the observed one if $T_{\mathrm{dec}}$ is around few $\mathrm{GeV}$.

The modulated decay temperature $T_{\mathrm{dec}} \propto \epsilon^{-1 / 2}$ produces the baryon isocurvature perturbation,

$$
S_{\mathrm{B}}=-\frac{5}{2} \frac{\delta \epsilon}{\epsilon}=5 \zeta,
$$

which is correlated with the curvature perturbation. This seems to be excluded by the CMB observations, but as we will see, the DM isocurvature perturbation naturally compensates the baryon isocurvature perturbation.

The compensation relies on the specific dependence of the baryon asymmetry on $T_{\text {dec }}$, which is altered if the inflaton-originated particles dominate the thermal bath of standard model particles at the electroweak phase transition (for which we find $S_{B}=4 \zeta$.) To avoid it, the Q-balls must dominate the Universe early enough, requiring large $m_{\phi}$,

$$
m_{\phi} \gtrsim 10 \mathrm{TeV}\left(\frac{|K|}{0.01}\right)^{5 / 13}\left(\frac{100}{g}\right)^{10 / 39},
$$

where we use Eq. ((6), (16)) to fix $\epsilon$. The bound is relaxed if the inflaton dominantly decays into particles decoupled from standard model particles. Still, the Universe must be Q-ball dominated when the temperature of the standard model bath produced from the Q-balls is around the electroweak scale, requiring

$$
m_{\phi} \gtrsim 200 \mathrm{GeV}\left(\frac{|K|}{0.01}\right)^{5 / 11}\left(\frac{100}{g}\right)^{10 / 33} .
$$

\section{DARK MATTER}

If DM abundance is established after the Q-balls decay, the DM isocurvature perturbation is absent. This should be the case if baryon asymmetry is also produced after the Q-balls decay. When baryon asymmetry is created by the Q-ball decay, the temperature at which the Q-balls decay is around a GeV scale, which is well after the freeze-out of the LSP, or that of the Next-to-LSP (NLSP) if the gravitino is the LSP. The thermal relic of DM is diluted by the entropy production from the Q-balls which dominate the Universe before they decay. The main source of the LSP is hence the nonthermal production from the Q-ball decay.

Suppose the annihilation of the LSP after the nonthermal production is negligible. This is the case if the bino is the LSP or the gravitino is the LSP and the NLSP quickly decays into the gravitino. The DM abundance is proportional to $T_{\mathrm{dec}}$, producing a DM isocurvature perturbation $S_{\mathrm{DM}}=-(\delta \epsilon / \epsilon) / 2=\zeta$. Together with the baryon isocurvature perturbation, this scenario predicts a matter isocurvature perturbation that is too large and is excluded by the CMB observations.

If the LSP is electroweak charged (i.e., wino or Higgsino-like) or is the gravitino but the NLSP does not decay into the gravitino quickly and is standard model gauge charged (any superpartners but a binolike one), the annihilation of the (N)LSP just after the production diminishes and determines the DM abundance $[65,66]$,

$$
\begin{aligned}
\frac{\rho_{\mathrm{DM}}}{s} & \left.\simeq m_{\mathrm{LSP}} \frac{H}{\langle\sigma v\rangle s}\right|_{T=T_{\mathrm{dec}}} \\
& \simeq 0.4 \mathrm{eV} \frac{3 \mathrm{GeV}}{T_{\mathrm{dec}}} \frac{m_{\mathrm{LSP}}}{0.7 \mathrm{TeV}}\left(\frac{m_{(\mathrm{N}) \mathrm{LSP}}}{0.7 \mathrm{TeV}}\right)^{2} \frac{0.01 / m_{(\mathrm{N}) \mathrm{LSP}}^{2}}{\langle\sigma v\rangle},
\end{aligned}
$$

which is valid for $T_{\text {dec }}$ below the freeze-out temperature of the (N)LSP. If the LSP is not the gravitino, the LSP should have a mass below TeV. In gravity mediation, if the LSP is the gravitino, the NSLP is not much heavier than the gravitino and the NLSP decays during the big bang nucleosynthesis. Such a case is excluded unless the sneutrino is the LSP [67]. In both cases, the resultant $\mathrm{DM}$ isocurvature perturbation is

$$
S_{\mathrm{DM}}=\frac{1}{2} \frac{\delta \epsilon}{\epsilon}=-\zeta,
$$

which is $-1 / 5$ of $S_{B}$. 


\section{COMPENSATED ISOCURVATURE PERTURBATIONS}

As DM is nearly 5 times more abundant than baryons, the matter isocurvature,

$$
S_{m}=\frac{\Omega_{\mathrm{DM}}}{\Omega_{m}} S_{\mathrm{DM}}+\frac{\Omega_{B}}{\Omega_{m}} S_{B}
$$

nearly vanishes according to Eqs. (17) and (21). That is, the isocurvature perturbations are compensated with each other and are less constrained by CMB observations [68]. In our model, the compensation is a natural consequence of the dependence of the baryon asymmetry and the DM abundance on $T_{\mathrm{dec}}$, while the compensation in the literatures $[68,69]$ requires tuning of model parameters.

The bound $\mathcal{P}_{S_{m}}<0.001 \mathcal{P}_{\zeta}$ [52] is satisfied for $4.8<$ $\Omega_{\mathrm{DM}} / \Omega_{B}<5.2$, which is consistent with the measurement of the abundances by Planck [2] within the $2 \sigma$ level. Future observations can determine the ratio $\Omega_{\mathrm{DM}} / \Omega_{B}$ with an absolute uncertainty of 0.02 [70] and probe $\mathcal{P}_{S_{m}} / \mathcal{P}_{\zeta}$ as small as 0.0002 [71]. The scenario can be tested by the future observations unless $4.9<\Omega_{\mathrm{DM}} / \Omega_{B}<5.1$.

The prediction is altered if there exists another subdominant component of DM. A well-motivated example is the QCD axion [72-75], which solves the strong $C P$ problem [76]. If the oscillation of the axion begins at $T>T_{\mathrm{dec}}$, the axion DM abundance depends on a positive power of $T_{\mathrm{dec}}$. This produces a DM isocurvature perturbation correlated with the curvature perturbation and reduces the matter isocurvature perturbation. Although the matter isocurvature perturbation is no longer uniquely predicted, one may check the consistency of the scenario once the decay constant and the abundance of the QCD axion are measured.

\section{LEPTON ASYMMETRY}

The L-balls decay into SM particles and create large and negative lepton asymmetry,

$$
\frac{n_{L_{i}}}{s} \simeq-3 \times 10^{-4}\left|q_{L_{i}}\right| \frac{T_{\mathrm{dec}}}{4 \mathrm{GeV}} \frac{10 \mathrm{TeV}}{m_{\phi}},
$$

where $i$ is the generation index and $q_{L_{i}}$ is the $i$ th generation lepton number of the Q-balls. Assuming that the asymmetry is equally distributed among three generations by neutrino oscillation [77-79], the bound $\left|n_{L_{i}} / s\right| \lesssim 0.01$ [80] requires $m_{\phi} \gtrsim 100 \mathrm{GeV}$.

If $m_{\phi}$ is $O(100) \mathrm{GeV}$, large negative lepton asymmetry increases the abundance of antielectron neutrinos and hence, the neutron-proton ratio, leading to a larger helium abundance produced by the big bang nucleosynthesis. Since the recombination is more effective, more baryon asymmetry is required to fit the $\mathrm{CMB}$ spectrum, helping the compensation. It will be interesting to check if future observations can probe this scenario. Note that $m_{\phi}=O(100) \mathrm{GeV}$ violates the bound (18) and requires that the inflaton decays into a hidden sector.

The lepton asymmetry increases the effective number of neutrinos $N_{\text {eff }}$ [81],

$$
\Delta N_{\mathrm{eff}}=\frac{3698 \pi^{2}}{105} \sum_{i}\left(\frac{n_{L_{i}}}{s}\right)^{2}
$$

Taking into account the above mentioned upper bound, $\Delta N_{\text {eff }} \ll 1$. If a particular combination of $L_{i}$ is produced, however, $\left|L_{e}\right| \ll\left|L_{\mu, \tau}\right|$, and $\Delta N_{\text {eff }}$ may be large [82]. For $T_{\text {dec }} / m_{\phi}=0.01, \Delta N_{\text {eff }} \simeq 0.02$, which is within the sensitivity of the CMB-S4 experiment [83,84].

\section{LSP AND SCALAR MASSES}

Figure 1 summarizes the constraints and predictions in our scenario. Allowing $|K| \in\left(10^{-4}, 0.1\right)$ and $\epsilon \in(0.01,1)$, we find that the resulting baryon asymmetry cannot be consistent with the observed value in the blue-shaded regions. The green and gray shaded regions are excluded since the Q-balls dominate the Universe after electroweak phase transition [Eq. (19)] and $\phi_{\mathrm{osc}}>m_{\mathrm{pl}}$, respectively. Here, we take $\epsilon=0.1$. The orange line is a lower bound on $m_{\phi}$ when the inflaton dominantly reheats the SM particles [Eq. (18)]. The measures above the plot shows the prediction on the masses of the pure wino LSP $\left(m_{\tilde{w}}\right)$ and the pure Higgsino LSP $\left(m_{\tilde{h}}\right)$. The red dashed lines show the lower bound on $m_{\phi}$ below which $m_{\phi}<m_{\tilde{w}, \tilde{h}}$. We note, however, that $m_{\phi}$ is the soft mass of the flat direction at a high energy scale and hence, is smaller than the one at the TeV scale; $K$ in Eq. (4) is negative. The red dashed lines should be regarded as conservative bounds. We predict $m_{\tilde{w}} \lesssim 1 \mathrm{TeV}$ for the pure-wino LSP and $m_{\tilde{h}} \lesssim 0.5 \mathrm{TeV}$ for

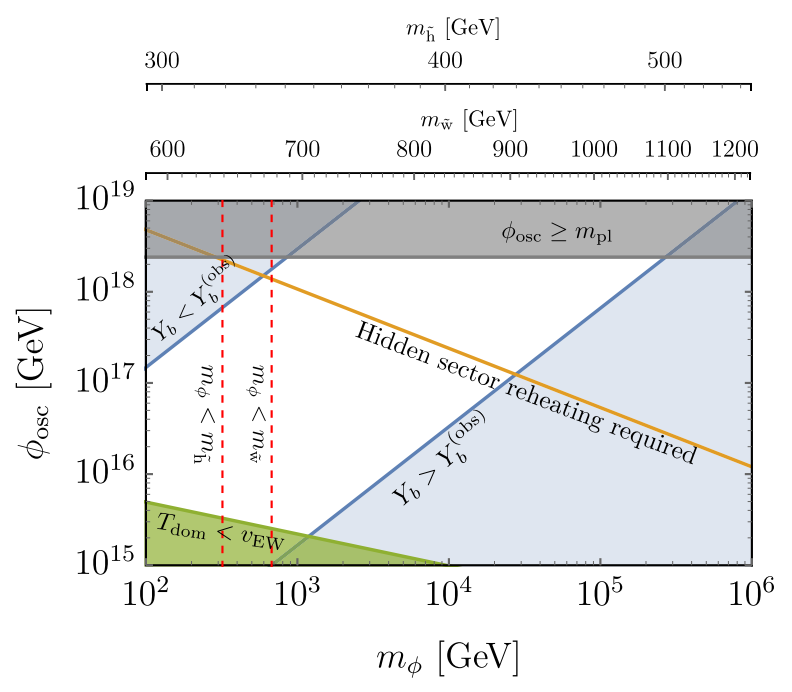

FIG. 1. Exclusion plot in $\phi_{\mathrm{osc}}-m_{\phi}$ plane. The measures above the figure represents the predictions of masses of the pure-wino $\operatorname{LSP}\left(m_{\tilde{w}}\right)$ and the pure-Higgsino LSP $\left(m_{\tilde{h}}\right)$. 
the pure-Higgsino LSP. With non-negligible mixing among neutralinos, the predicted mass becomes even smaller because of a smaller annihilation cross section.

The predicted LSP mass is below the prediction of the thermal freeze-out mechanism, $m_{\tilde{w}} \simeq 2.7 \mathrm{TeV}$ and $m_{\tilde{h}} \simeq$ $1 \mathrm{TeV}[85,86]$. The Large Hadron Collider (LHC) already excludes $m_{\tilde{w}}<474 \mathrm{GeV}$ and $m_{\tilde{h}}<175 \mathrm{GeV}$ [87]. The projected sensitivity of the high-luminosity LHC is $m_{\tilde{w}}<$ $900 \mathrm{GeV}$ and $m_{\tilde{h}}<300 \mathrm{GeV}$ [88], probing some of our parameter space. The 27-TeV upgrade of the LHC [89] can reach $m_{\tilde{w}}<2.1 \mathrm{TeV}$ and $m_{\tilde{h}}<600 \mathrm{GeV}$ [88], covering the whole parameter space.

The observation of gamma-rays by the Fermi-LAT Collaboration constraints the annihilation cross section of DM. Observations of dwarf spheroidal galaxies excluded $m_{\tilde{w}}<390 \mathrm{GeV}$ and $2.14 \mathrm{TeV}<m_{\tilde{w}}<2.53 \mathrm{TeV}$ at 95\% C.L. [90]. Ten years of data taking by the FermiLAT will probe $m_{\tilde{w}}<810 \mathrm{GeV}$ and $1.9 \mathrm{TeV}<m_{\tilde{w}}<$ $2.7 \mathrm{TeV}$ [91], covering our parameter space with $m_{\phi}<10 \mathrm{TeV}$. See [92] for the future prospect of the observation of the center of the Galaxy, although it suffers from the uncertainty of the DM distribution.

\section{DISCUSSION}

Our scenario has the following beyond- $\Lambda \mathrm{CDM}$ parameters: (1) local non-Gaussianity $f_{\mathrm{NL}}=5 / 3$ and (2) baryon and DM isocurvature perturbations nearly compensating with each other. If the inflaton dominantly decays into a hidden sector, (3) lepton asymmetry and (4) effective number of neutrinos can be sizable. The LSP mass must be below $\mathrm{TeV}$ and be Wino- or Higgsino-like unless the gravitino is the LSP. The LSP can be searched for by future colliders and indirect detection experiments.

In this paper, we assume that only L-balls are formed. One may naively expect that in $S U(5)$ unified theories, when a flat direction to form L-balls has a large field value,
$S U(5)$ partners of the flat direction also have large field values to form B-balls. This is not necessarily the case. For example, we may consider the $\bar{d}_{1} L_{1} Q_{2}$ flat direction, where the subscripts are generation indices. The $S U(5)$ partners $L_{1} L_{1} \bar{e}_{2}$ and $\bar{d}_{1} \bar{d}_{1} \bar{u}_{2}$ identically vanish. We may also use the $L H_{u}$ direction if the lightest neutrino is nearly massless. The $S U(5)$ partner of the direction involves a heavy colored Higgs boson and cannot be excited. Other possibilities include embedding of quarks and leptons to different multiplets of $S U(5)$, or orbifold GUTs [93,94].

We comment on production of primordial black holes (PBHs). References [95-98] argued that the density fluctuations of Q-balls grow rapidly enough to form $\mathrm{PBHs}$ during the Q-ball dominated era. However, their assumption on the evolution of the density fluctuations may be too optimistic as is briefly commented in [98]. Since the estimations of the detailed mass spectrum in the literature are not yet reliable, we only estimate the typical PBH mass. The growth of the density fluctuations is the most efficient for the mode that enters the horizon at the beginning of the Q-ball domination. The typical PBH mass is expected to be the horizon mass at that time,

$$
M_{\mathrm{PBH}} \sim 10^{20} \mathrm{~g}\left(\frac{|K|}{0.01}\right)^{3}\left(\frac{g}{100}\right)\left(\frac{m_{\phi}}{1 \mathrm{TeV}}\right)^{-31 / 5}
$$

which is in the range $10^{10-25} \mathrm{~g}$. Various astrophysical constraints and searches exist for this mass range [99].

\section{ACKNOWLEDGMENTS}

KH thanks Fuminobu Takahashi and Benjamin Wallisch for useful discussion. This work was supported in part by the Director, Office of Science, Office of High Energy and Nuclear Physics, of the US Department of Energy under Contract No. DE-SC0009988 (K. H.).
[1] C. L. Bennett et al. (WMAP Collaboration), Astrophys. J. Suppl. Ser. 208, 20 (2013).

[2] N. Aghanim et al. (Planck Collaboration), Astron. Astrophys. 641, A6 (2020).

[3] V. F. Mukhanov and G. V. Chibisov, Pis'ma Zh. Eksp. Teor. Fiz. 33, 549 (1981) [JETP Lett. 33, 532 (1981)].

[4] S. W. Hawking, Phys. Lett. 115B, 295 (1982).

[5] A. A. Starobinsky, Phys. Lett. 117B, 175 (1982).

[6] A. H. Guth and S. Y. Pi, Phys. Rev. Lett. 49, 1110 (1982).

[7] J. M. Bardeen, P. J. Steinhardt, and M. S. Turner, Phys. Rev. D 28, 679 (1983).

[8] K. Enqvist and M. S. Sloth, Nucl. Phys. B626, 395 (2002).

[9] D. H. Lyth and D. Wands, Phys. Lett. B 524, 5 (2002).
[10] T. Moroi and T. Takahashi, Phys. Lett. B 522, 215 (2001); 539, 303(E) (2002).

[11] S. Dimopoulos, S. Raby, and F. Wilczek, Phys. Rev. D 24, 1681 (1981).

[12] S. Dimopoulos and H. Georgi, Nucl. Phys. B193, 150 (1981).

[13] N. Sakai, Z. Phys. C 11, 153 (1981).

[14] L. E. Ibanez and G. G. Ross, Phys. Lett. 105B, 439 (1981).

[15] M. B. Einhorn and D. R. T. Jones, Nucl. Phys. B196, 475 (1982).

[16] W. J. Marciano and G. Senjanovic, Phys. Rev. D 25, 3092 (1982).

[17] E. Witten, Nucl. Phys. B188, 513 (1981). 
[18] H. Pagels and J. R. Primack, Phys. Rev. Lett. 48, 223 (1982).

[19] H. Goldberg, Phys. Rev. Lett. 50, 1419 (1983).

[20] L. Maiani, Conf. Proc. C7909031, 1 (1979).

[21] M. J. G. Veltman, Acta Phys. Pol. B 12, 437 (1981).

[22] R. K. Kaul, Phys. Lett. 109B, 19 (1982).

[23] K. Enqvist, S. Kasuya, and A. Mazumdar, Phys. Rev. Lett. 90, 091302 (2003).

[24] K. Enqvist, A. Jokinen, S. Kasuya, and A. Mazumdar, Phys. Rev. D 68, 103507 (2003).

[25] S. Kasuya, M. Kawasaki, and F. Takahashi, Phys. Lett. B 578, 259 (2004).

[26] K. Hamaguchi, M. Kawasaki, T. Moroi, and F. Takahashi, Phys. Rev. D 69, 063504 (2004).

[27] J. McDonald, Phys. Rev. D 69, 103511 (2004).

[28] A. Riotto and F. Riva, Phys. Lett. B 670, 169 (2008).

[29] M. Dine, L. Randall, and S. D. Thomas, Phys. Rev. Lett. 75, 398 (1995).

[30] M. Dine, L. Randall, and S. D. Thomas, Nucl. Phys. B458, 291 (1996).

[31] K. Harigaya, M. Ibe, M. Kawasaki, and T. T. Yanagida, J. Cosmol. Astropart. Phys. 11 (2015) 003.

[32] K. Mukaida, K. Nakayama, and M. Takimoto, J. Cosmol. Astropart. Phys. 06 (2014) 013.

[33] D. H. Lyth, C. Ungarelli, and D. Wands, Phys. Rev. D 67, 023503 (2003).

[34] S. R. Coleman, Nucl. Phys. B262, 263 (1985); B269, 744 (E) (1986).

[35] A. Kusenko, Phys. Lett. B 404, 285 (1997).

[36] A. Kusenko, Phys. Lett. B 405, 108 (1997).

[37] A. Kusenko and M. E. Shaposhnikov, Phys. Lett. B 418, 46 (1998).

[38] K. Enqvist and J. McDonald, Phys. Lett. B 425, 309 (1998).

[39] K. Enqvist and J. McDonald, Nucl. Phys. B538, 321 (1999).

[40] S. Kasuya and M. Kawasaki, Phys. Rev. D 61, 041301 (2000).

[41] M. Dine and A. Kusenko, Rev. Mod. Phys. 76, 1 (2003).

[42] A. G. Cohen, S. R. Coleman, H. Georgi, and A. Manohar, Nucl. Phys. B272, 301 (1986).

[43] V. A. Kuzmin, V. A. Rubakov, and M. E. Shaposhnikov, Phys. Lett. 155B, 36 (1985).

[44] I. Affleck and M. Dine, Nucl. Phys. B249, 361 (1985).

[45] T. Hiramatsu, M. Kawasaki, and F. Takahashi, J. Cosmol. Astropart. Phys. 06 (2010) 008.

[46] S. Kasuya and M. Kawasaki, Phys. Rev. D 62, 023512 (2000).

[47] F. Doddato and J. McDonald, J. Cosmol. Astropart. Phys. 06 (2011) 008.

[48] A. Kamada, M. Kawasaki, and M. Yamada, Phys. Lett. B 719, 9 (2013).

[49] M. Sasaki and E. D. Stewart, Prog. Theor. Phys. 95, 71 (1996).

[50] D. Wands, K. A. Malik, D. H. Lyth, and A. R. Liddle, Phys. Rev. D 62, 043527 (2000).

[51] D. H. Lyth, K. A. Malik, and M. Sasaki, J. Cosmol. Astropart. Phys. 05 (2005) 004.

[52] Y. Akrami et al. (Planck Collaboration), Astrophys. Space Sci. 364, 69 (2019).

[53] G. Dvali, A. Gruzinov, and M. Zaldarriaga, Phys. Rev. D 69, 023505 (2004).
[54] L. Kofman, arXiv:astro-ph/0303614.

[55] D. H. Lyth and A. Riotto, Phys. Rep. 314, 1 (1999).

[56] D. H. Lyth, Phys. Rev. Lett. 78, 1861 (1997).

[57] M. Alvarez et al., arXiv:1412.4671.

[58] O. Doré et al., arXiv:1412.4872.

[59] J. B. Muñoz, Y. Ali-Haïmoud, and M. Kamionkowski, Phys. Rev. D 92, 083508 (2015).

[60] T. Multamaki and I. Vilja, Phys. Lett. B 535, 170 (2002).

[61] A. G. Cohen, D. B. Kaplan, and A. E. Nelson, Phys. Lett. B 245, 561 (1990).

[62] M. Carena, G. Nardini, M. Quiros, and C. E. M. Wagner, Nucl. Phys. B812, 243 (2009).

[63] T. Asaka and M. Shaposhnikov, Phys. Lett. B 620, 17 (2005).

[64] M. D’Onofrio, K. Rummukainen, and A. Tranberg, Phys. Rev. Lett. 113, 141602 (2014).

[65] M. Fujii and K. Hamaguchi, Phys. Rev. D 66, 083501 (2002).

[66] M. Fujii, K. Hamaguchi, and T. Yanagida, Phys. Rev. D 65, 115012 (2002).

[67] M. Kawasaki, K. Kohri, T. Moroi, and A. Yotsuyanagi, Phys. Rev. D 78, 065011 (2008).

[68] C. Gordon and A. Lewis, Phys. Rev. D 67, 123513 (2003).

[69] K. Harigaya, T. Hayakawa, M. Kawasaki, and S. Yokoyama, J. Cosmol. Astropart. Phys. 10 (2014) 068.

[70] D. Baumann, D. Green, and B. Wallisch, J. Cosmol. Astropart. Phys. 08 (2018) 029.

[71] F. Finelli et al. (CORE Collaboration), J. Cosmol. Astropart. Phys. 04 (2018) 016.

[72] R. D. Peccei and H. R. Quinn, Phys. Rev. Lett. 38, 1440 (1977).

[73] R. D. Peccei and H. R. Quinn, Phys. Rev. D 16, 1791 (1977).

[74] S. Weinberg, Phys. Rev. Lett. 40, 223 (1978).

[75] F. Wilczek, Phys. Rev. Lett. 40, 279 (1978).

[76] G. 't Hooft, Phys. Rev. Lett. 37, 8 (1976).

[77] A. D. Dolgov, S. H. Hansen, S. Pastor, S. T. Petcov, G. G. Raffelt, and D. V. Semikoz, Nucl. Phys. B632, 363 (2002).

[78] Y. Y. Y. Wong, Phys. Rev. D 66, 025015 (2002).

[79] G. Mangano, G. Miele, S. Pastor, O. Pisanti, and S. Sarikas, Phys. Lett. B 708, 1 (2012).

[80] I. M. Oldengott and D. J. Schwarz, Europhys. Lett. 119, 29001 (2017).

[81] J. Lesgourgues and S. Pastor, Phys. Rev. D 60, 103521 (1999).

[82] G. Barenboim, W. H. Kinney, and W.-I. Park, Phys. Rev. D 95, 043506 (2017).

[83] W. L. K. Wu, J. Errard, C. Dvorkin, C. L. Kuo, A. T. Lee, P. McDonald, A. Slosar, and O. Zahn, Astrophys. J. 788, 138 (2014).

[84] K. N. Abazajian et al. (CMB-S4 Collaboration), arXiv: 1610.02743 .

[85] J. Hisano, S. Matsumoto, M. Nagai, O. Saito, and M. Senami, Phys. Lett. B 646, 34 (2007).

[86] M. Cirelli, A. Strumia, and M. Tamburini, Nucl. Phys. B787, 152 (2007).

[87] A. M. Sirunyan et al. (CMS Collaboration), Phys. Lett. B 806, 135502 (2020).

[88] T. Han, S. Mukhopadhyay, and X. Wang, Phys. Rev. D 98, 035026 (2018). 
[89] A. Abada et al. (FCC Collaboration), Eur. Phys. J. Special Topics 228, 1109 (2019).

[90] M. Ackermann et al. (Fermi-LAT Collaboration), Phys. Rev. D 89, 042001 (2014).

[91] B. Bhattacherjee, M. Ibe, K. Ichikawa, S. Matsumoto, and K. Nishiyama, J. High Energy Phys. 07 (2014) 080.

[92] L. Rinchiuso, N. L. Rodd, I. Moult, E. Moulin, M. Baumgart, T. Cohen, T. R. Slatyer, I. W. Stewart, and V. Vaidya, Phys. Rev. D 98, 123014 (2018).

[93] Y. Kawamura, Prog. Theor. Phys. 105, 999 (2001).
[94] L. J. Hall and Y. Nomura, Phys. Rev. D 64, 055003 (2001).

[95] E. Cotner and A. Kusenko, Phys. Rev. Lett. 119, 031103 (2017).

[96] E. Cotner and A. Kusenko, Phys. Rev. D 96, 103002 (2017).

[97] E. Cotner, A. Kusenko, and V. Takhistov, Phys. Rev. D 98, 083513 (2018).

[98] E. Cotner, A. Kusenko, M. Sasaki, and V. Takhistov, J. Cosmol. Astropart. Phys. 10 (2019) 077.

[99] B. Carr, K. Kohri, Y. Sendouda, and J. Yokoyama, arXiv: 2002.12778 . 